\title{
EXTENSION OF THE RAUCH COMPARISON THEOREM TO SUBMANIFOLDS
}

\author{
F. W. WARNER(1)
}

1. Introduction. The Rauch comparison theorem yields a metric comparison of the lengths of Jacobi fields along geodesics in different Riemannian manifolds under suitable initial conditions and suitable hypotheses on the curvatures and on the nonexistence of conjugate points. Part of the initial condition is that the Jacobi fields should vanish at the initial points. In this paper we show how Rauch's theorem and proof extend to Jacobi fields satisfying more general initial conditions; namely, to Jacobi fields associated with submanifolds. Berger has given such an extension in [2] for the case in which the submanifolds are themselves geodesics. For more general submanifolds the initial conditions will involve the second fundamental forms; and so in the comparison theorem, one needs an additional hypothesis comparing the second fundamental forms. Instead of conjugate points, one is now concerned with focal points. Several new factors enter. One is that one has to apply, in certain cases, some special boundary conditions in order to get a comparison, and another is that the comparison generally does not hold as far as the first focal point in contrast to the Rauch case where the comparison holds as far as the first conjugate point.

In $\$ 2$ we review some of the basic geometry of submanifolds and give a precise statement of the Rauch comparison theorem. In $\$ 3$ we give a formal setup and proof of the comparison theorem which we then apply to submanifolds in $\$ 4$.

2. Preliminaries. We refer the reader to [1] or [3] for details and proofs of the material summarized in this section. Unless we specify otherwise, we assume all manifolds and maps to be differentiable of class $C^{\infty}$. By a differentiable map with domain a closed interval $[a, b]$ of the real line we mean one which can be extended to be differentiable on an open neighborhood of the interval. A piecewise differentiable map on $[a, b]$ is one for which there is a partition

$$
a=\alpha_{0}<\alpha_{1}<\cdots<\alpha_{n}=b
$$

of $[a, b]$ such that the map is differentiable on each $\left[\alpha_{i}, \alpha_{i+1}\right]$.

Let $M$ be a $d$-dimensional Riemannian manifold with $d \geqq 2$. We denote the

Received by the editors September 10, 1965 .

(1) This work was supported in part by National Science Foundation Grant number GP-1610 and GP-1611. 
tangent space to $M$ at $m$ by $M_{m}$ and the inner product of tangent vectors $x$ and $y$ in $M_{m}$ by $\langle x, y\rangle$. The length or norm of $x$ is denoted by $\|x\|$. Let $K$ be a $p$-dimensional Riemannian submanifold of $M$ passing through the point $m$ of $M$ with $0 \leqq p \leqq d-1$. Let $\sigma(t), 0 \leqq t \leqq b$, be a geodesic in $M$ parametrized by arc length with initial point $m$ and initial tangent $\sigma_{*}(0)$ orthogonal to the tangent space $K_{m}$ of the submanifold at $m$. The second fundamental form assigns to the normal vector $\sigma_{*}(0)$ a symmetric linear transformation $S_{\sigma_{*}(0)}$ on $K_{m}$. Let $\mathscr{L}\left(\sigma, t_{0}, K\right)$ denote the linear space of all piecewise smooth vector fields along $\sigma \mid\left[0, t_{0}\right]$ whose values are everywhere orthogonal to the tangent vector $\sigma_{*}$ of $\sigma$ and whose initial value is an element of $K_{m} . \mathscr{L}\left(\sigma, t_{0}, 0\right)$ is the subspace of $\mathscr{L}\left(\sigma, t_{0}, K\right)$ consisting of those vector fields which vanish at $m$.

A smooth vector field $Y(t)$ along $\sigma$ is called a Jacobi field if it satisfies the Jacobi equation:

$$
\left(\nabla^{2} Y\right)(t)+R_{t} Y(t)=0 .
$$

$(\nabla Y)(t)$ means the covariant derivative of $Y$ along $\sigma$ with respect to $\sigma_{*}(t) . R_{t}$ is a symmetric linear transformation on $M_{\sigma(t)}$ defined as follows in terms of the curvature tensor:

$$
R_{t} Y(t)=R\left(Y(t), \sigma_{*}(t)\right) \sigma_{*}(t) .
$$

A Jacobi field $Y \in \mathscr{L}(\sigma, b, K)$ is called a $K$-Jacobi field if it satisfies the boundary condition:

$$
S_{\sigma_{*}(0)} Y(0)-(\nabla Y)(0) \in\left(K_{m}\right)^{\perp},
$$

where $\perp$ means orthogonal complement in $M_{m}$. Geometrically, the $K$-Jacobi fields are precisely those which arise as the base vector fields $d \alpha(\partial / \partial v)(t, 0)$ of mappings $\alpha(t, v)$ of rectangles into $M$ with longitudinal curves $\alpha$ ( $v=$ constant) geodesics emanating orthogonally from $K$ and parametrized by arc length. We call a $K$-Jacobi field $Y$ along $\sigma$ a strong $K$-Jacob field if it satisfies the strong boundary condition:

$$
S_{\sigma_{*}(0)} Y(0)=(\nabla Y)(0) \text {. }
$$

The set of $K$-Jacobi fields along $\sigma$ forms a $(d-1)$-dimensional linear space and spans the orthogonal complement to $\sigma_{*}(t)$ in $M_{\sigma(t)}$ for $0<t$ prior to the first focal point on $\sigma$. A focal point on $\sigma$ is a point $\sigma(t), t \neq 0$, at which a nontrivial $K$-Jacobi field along $\sigma$ vanishes.

In the case when $K=m$, the set of $K$-Jacobi fields along $\sigma$ is the set Jacobi fields in $\mathscr{L}(\sigma, b, 0)$, and focal points are also called conjugate points.

The index form $I_{\left(t_{0}, K\right)}$ is a symmetric bilinear form on $\mathscr{L}\left(\sigma, t_{0}, K\right)$ defined as follows:

$$
I_{\left(t_{0}, K\right)}(X, Y)=\left\langle S_{\sigma^{*}(0)} X(0), Y(0)\right\rangle+\int_{0}^{t_{0}}\left(\langle\nabla X, \nabla Y\rangle-\left\langle R_{t} X, Y\right\rangle\right) d t
$$


If $X \in \mathscr{L}\left(\sigma, t_{0}, K\right)$ and $X(t) \neq 0$, the sectional curvature $K(X)$ of the 2-plane in $M_{\sigma(t)}$ spanned by $X(t)$ and $\sigma_{*}(t)$ is equal to $\left\langle R_{t} X, X\right\rangle /\langle X, X\rangle$. If we define $K(X)$ to be 0 whenever $X$ vanishes, then

$$
I_{\left(t_{0}, K\right)}(X, X)=\left\langle S_{\sigma_{*}(0)} X(0), X(0)\right\rangle+\int_{0}^{t_{0}}(\langle\nabla X, \nabla X\rangle-K(X)\langle X, X\rangle) d t .
$$

When there is no confusion we let $I=I_{\left(t_{0}, K\right)}$. If $Y$ is a $K$-Jacobi field in $\mathscr{L}\left(\sigma, t_{0}, K\right)$,

$$
I(Y, Y)=\left\langle(\nabla Y)\left(t_{0}\right), Y\left(t_{0}\right)\right\rangle=(1 / 2)\langle Y, Y\rangle^{\prime}\left(t_{0}\right) .
$$

One of the basic properties of Jacobi fields is that they minimize the index form prior to focal points in the following sense.

Minimization Theorem 2.1. Let $X \in \mathscr{L}(\sigma, b, K)$. Assume there are no focal points on $\sigma$. Then there exists a unique $K$-Jacobi field $Y$ such that $Y(b)=X(b)$ and

$$
I(X, X) \geqq I(Y, Y)
$$

with equality if and only if $X=Y$.

COROLlaRY 2.2. If there are no focal points on $\sigma, I$ is positive definite on the subspace of $\mathscr{L}(\sigma, b, K)$ consisting of those vector fields vanishing at $\sigma(b)$.

If, however, there are focal points on $\sigma$, then $I$ is not positive definite on the subspace of $\mathscr{L}(\sigma, b, K)$ consisting of those vector fields vanishing at $\sigma(b)$. Indeed there exists a nontrivial $K$-Jacobi field $Y$ vanishing at some $t_{0} \leqq b$. If we extend $Y$ to be 0 from $t_{0}$ to $b$ then $I(Y, Y)=0$. In particular, in the case $K=m$, if there are no conjugate points on $\sigma, I$ is positive definite on the subspace of $\mathscr{L}(\sigma, b, 0)$ consisting of those vector fields vanishing at $\sigma(b)$; and if there are conjugate points on $\sigma, I$ is not positive definite on this subspace of $\mathscr{L}(\sigma, b, 0)$. Since $\mathscr{L}(\sigma, b, 0) \subset \mathscr{L}(\sigma, b, K)$ we have the

COROLlaRY 2.3. The first focal point on the geodesic $\sigma$ for any submanifold $K$ passing through $\sigma(0)$ with $\sigma_{*}(0) \in\left(K_{\sigma(0)}\right)^{\perp}$ occurs at least as soon as the first conjugate point to $\sigma(0)$ along $\sigma$.

Let $M, K$ and $\sigma$ be as above. We distinguish another such setup by the presuperscript '. So ' $M$ is a ' $d$-dimensional Riemannian manifold, ' $d \geqq 2$, ' $K$ a ' $p$ dimensional Riemannian submanifold of ' $M$ with $0 \leqq '^{\prime} p \leqq ' d-1$, ' $m$ a point of $' K,{ }^{\prime} \sigma(t)$ a geodesic in ' $M$ parametrized by arc length with domain the interval $[0, b]$, initial point ' $m$ and initial derivative ${ }^{\prime} \sigma_{*}(0) \in\left({ }^{\prime} K_{\prime m}\right){ }^{\perp}$.

The Rauch comparison theorem [5], [6], [3] deals with the case in which $K$ and ' $K$ are both points and gives a comparison of the length of $K$ and ' $K$-Jacobi fields along $\sigma$ and ' $\sigma$ under suitable curvature and nonconjugacy hypotheses. 
Rauch Comparison Theorlm 2.4. Let $d={ }^{\prime} d$. Assume there are no conjugate points on ' $\sigma$ and assume that for each $t \in[0, b]$, for all 2-planes $P \subset M_{\sigma(t)}$ containing $\sigma_{*}(t)$ and all 2-planes $Q \subset{ }^{\prime} M^{\prime} \sigma(t)$ containing ${ }^{\prime} \sigma_{*}(t)$ the sectional curvatures satisfy:

$$
K(P) \leqq K(Q) .
$$

Let $X$ and $Y$ be Jacobi fields in $\mathscr{L}(\sigma, b, 0)$ and $\mathscr{L}\left({ }^{\prime} \sigma, b, 0\right)$ respectively, such tha $\|(\nabla X)(0)\|=\|(\nabla Y)(0)\|$. Then

$$
\|X(t)\| \geqq\|Y(t)\| \quad \text { for all } t \in[0, b] .
$$

Berger has indicated in [2] how Rauch's proof may be extended to give a comparison of certain $K$ and ' $K$-Jacobi fields in the case where both $K$ and ' $K$ are geodesics. It is our purpose here to show how Rauch's proof may be extended to the case of general submanifolds $K$ and ' $K$. One requires in the general case an additional hypothesis comparing the second fundamental forms $S_{\sigma_{*}(0)}$ and $S_{\sigma_{*}(0)}$. Several other new factors enter in the general case in that one has to make suitable boundary restrictions on the Jacobi fields to get ones which can be compared, and in certain cases the comparison can be carried beyond the first focal point whereas in others the comparison does not hold as far as the first focal point. We also would like to have a comparison when the dimensions $d$ and ' $d$ are not equal. In the case ' $d<d$, one has to formally increase the number of variables in the Jacobi equation along ' $\sigma$. To do this we find it most convenient to separate the geometric application from the result on differential equations involved. This we do in the next section where we treat formally the comparison theorem. In $\$ 4$ we apply this to submanifolds.

3. The comparison theorem. We first formalize the geometric setup of $\S 2$ essentially by choosing a parallel frame along $\sigma$ and identifying all the tangent spaces $M_{\sigma(t)}$ along $\sigma$ with $M_{m}$. Actually, since we are only interested in Jacobi fields orthogonal to $\sigma_{*}$ along $\sigma$, we only consider the subspace of $M_{\sigma(t)}$ orthogonal to $\sigma_{*}(t)$. For the most part we use similar notation and terminology. One difference, however, is that here we will only require the coefficients in the Jacobi operator to be continuous, and hence the solutions of the Jacobi equation will be differentiable of class $C^{2}$.

Again let $d$ be an integer $\geqq 2$. Let $V$ be a $(d-1)$-dimensional real vector space with an inner product $\langle$,$\rangle . Let t \rightarrow R_{t}$ be a continuous mapping of $[0, b]$ into the symmetric linear transformations on $V$. Let $K$ be a subspace of $V$ of dimension $p$ with $0 \leqq p \leqq d-1$, and $S$ a symmetric linear transformation on $K$. Let $\mathscr{L}\left(V, t_{0}, K\right)$ denote the linear space of piecewise $C^{2}$ curves $Y(t)$ in $V$ with domain $\left[0, t_{0}\right]$ and initial value $Y(0) \in K$. We call a $C^{2}$ curve $Y$ in $V$ a Jacobi field if it satisfies the Jacobi equation:

$$
\left(\nabla^{2} Y\right)(t)+R_{t} Y(t)=0
$$


Here $\nabla$ denotes the ordinary derivative of $V$ valued functions with domain an open subset of the real line. If $Y$ is a differentiable curve in $V, \nabla Y$ is defined and is again a curve in $V$. A Jacobi field $Y \in \mathscr{L}\left(V, t_{0}, K\right)$ is a $K$-Jacobi field if

$$
S(Y(0))-(\nabla Y)(0) \in K^{\perp} \text {. }
$$

Let $\mathscr{V}$ denote the setup consisting of $\left(V, R_{t}, K, S\right)$. A real number $t_{0} \in(0, b]$ is a focal point of $\mathscr{V}$ if there exists a nontrivial $K$-Jacobi field $Y$ such that $Y\left(t_{0}\right)=0$. In the case $K=\{0\}$, focal points are also called conjugate points. The index form $I_{\left(t_{0}, K\right)}$ is the symmetric bilinear form on $\mathscr{L}\left(V, t_{0}, K\right)$ defined by:

$$
I_{\left(t_{0}, K\right)}(X, Y)=\langle S X(0), Y(0)\rangle+\int_{0}^{t_{0}}\left(\langle\nabla X, \nabla Y\rangle-\left\langle R_{t} X, Y\right\rangle\right) d t .
$$

It can be checked that under our new differentiability assumptions the minimization theorem holds as before.

Minimization THEOREM 3.1. Let $X \in \mathscr{L}(V, b, K)$ and assume there are no focal points of $\mathscr{V}$ on $(0, b]$. Then there exists a unique $K$-Jacobi field $Y$ such that $Y(b)=X(b)$ and

$$
I(X, X) \geqq I(Y, Y)
$$

with equality if and only if $X=Y$.

In our applications in $\S 4, V$ will be the subspace of $M_{\sigma(0)}$ orthogonal to $\sigma_{*}(0), K$ will be $K_{m}$ and $S$ will be $S_{\sigma_{*}(0)}$. Let $P_{t}$ denote parallel translation of $M_{\sigma(0)}$ to $M_{(t)}$ along $\sigma$. Then the symmetric linear transformation $R_{t}$ on $V$ will be $P_{t}^{-1} R_{t} P_{t}$, where the latter $R_{t}$ is the curvature transformation on $M_{\sigma(t)}$.

Now consider another such setup ' $\mathscr{V}=\left({ }^{\prime} V,{ }^{\prime} R_{t},{ }^{\prime} K,{ }^{\prime} S\right)$ where ' $V$ is of dimension $(' d-1) \geqq 1$, and ' $K$ is of dimension ' $p$ with $0 \leqq{ }^{\prime} p \leqq{ }^{\prime} d-1$. We first need the following comparison of the relative location of the first local points of $\mathscr{V}$ and ' $\mathscr{V}$.

THEOREM 3.2. Assume that if $p>0$ then also ' $p>0$. Assume for each $t \in[0, b]$ that the maximum eigenvalue of $R_{t}$ is less than or equal to the minimum eigenvalue of ' $R_{t}$. Assume the minimum eigenvalue of $S$ is greater than or equal to the maximum eigenvalue of ' $S$. Then if there are no focal points of ' $\mathscr{V}$ on $(0, b]$, there are no focal points of $\mathscr{V}$ on $(0, b]$.

REMARK. We do not distinguish the case where $p=0$ and ' $p \geqq 0$. Simply observe that in this case $S$ and the corresponding term in the index form for $\mathscr{V}$ vanish and there is no restriction on the eigenvalues of ' $S$ if ' $S$ exists.

Proof. Assume $t_{0} \in(0, b]$ is a focal point of $\mathscr{V}$. We show ' $\mathscr{V}$ has a focal point on $(0, b]$. Assume first that $d \leqq ' d$. There is a nontrivial $K$-Jacobi field $Y$ such that $Y\left(t_{0}\right)=0$. Extend $Y$ to be 0 from $t_{0}$ to $b$. Then $I_{(b, K)}(Y, Y)=0$. Let $e_{1}, \cdots, e_{d-1}$ be an orthonormal basis of $V$ such that the only nonzero component of $Y(0)$, if there is any, is in the $e_{1}$ direction. 


$$
Y(t)=\sum_{i=1}^{d-1} g_{i}(t) e_{i}
$$

Let $f_{1}, \cdots, f^{\prime} d-1$ be an orthonormal basis for ' $V$ with $f_{1} \in{ }^{\prime} K$ if ${ }^{\prime} K \neq\{0\}$. Define:

$$
Z(t)=\sum_{i=1}^{d-1} g_{i}(t) f_{i} .
$$

Then $Z(t) \in \mathscr{L}\left({ }^{\prime} V, b,{ }^{\prime} K\right)$. The maximum eigenvalue of $R_{t}$ is the maximum of $\left\langle R_{t} v, v\right\rangle$ as $v$ runs over the unit sphere in $V$ and similarly for the minimum. Therefore since $\|Y(t)\|=\|Z(t)\|$ for every $t$, our hypotheses imply

$$
\left\langle R_{t} Y, Y\right\rangle \leqq\left\langle R_{t} Z, Z\right\rangle \text {. }
$$

Similarly

$$
\langle S Y(0), Y(0)\rangle \geqq\left\langle{ }^{\prime} S Z(0), Z(0)\right\rangle \text {. }
$$

Hence

$$
0=I_{(b, K)}(Y, Y) \geqq I_{\left(b,{ }^{\prime} K\right)}(Z, Z) .
$$

Therefore ' $\mathscr{V}$ has a focal point on $(0, b]$ for otherwise, by the minimization theorem, $I_{\left(b,{ }^{\prime} K\right)}$ would be positive definite on the subspace of $\mathscr{L}\left({ }^{\prime} V, b,{ }^{\prime} K\right)$ consisting of those curves in ' $V$ vanishing at $b$.

Now consider the case ' $d<d$. Let $W$ be a $\left(d-{ }^{\prime} d\right)$-dimensional real inner product space and let

$$
\text { " } V={ }^{\prime} V \oplus W
$$

with the induced inner product on " $V$. Let $v(t)$ be the minimum eigenvalue of ' $R_{t}$. Then $v(t)$ is a continuous function of $t$. (Note that even if we had assumed initially that ' $R_{t}$ was to be differentiable of class $C^{\infty}$, we could at best conclude $v(t)$ is continuous.) Let

$$
\text { " } K={ }^{\prime} K \oplus\{0\} \subset " V
$$

and let " $R_{t}$ be the symmetric linear transformation on " $V$ and " $S$ the symmetric linear transformation on " $K$ defined by:

$$
{ }^{\prime \prime} R_{t}={ }^{\prime} R_{t} \oplus v(t) I
$$

and

$$
\text { " } S={ }^{\prime} S \oplus 0,
$$

where $I$ is the identity on $W$. Let " $\mathscr{V}=\left(" V, R_{t}, " K, " S\right)$. Then the hypotheses of the theorem are satisfied for $\mathscr{V}$ and " $\mathscr{V}$. Namely, if $p>0$ then " $p>0$, the maximum eigenvalue of $R_{t}$ is less than or equal to the minimum eigenvalue of " $R_{t}$, and the 
minimum eigenvalue of $S$ is greater than or equal to the maximum eigenvalue of " $S$. We need only show that there are no focal points of " $\mathscr{V}$ on $(0, b]$. Then since $d=" d$, it follows from the first part of the proof that there are no focal points of $\mathscr{V}$ on $(0, b]$. Because of the way " $\mathscr{V}$ was constructed, the first focal point of " $\mathscr{V}$ is either the first focal point of ' $\mathscr{V}$ or the first conjugate point for $(W, v(t) I,\{0\}, 0)$. By assumption, there are no focal points for ' $\mathscr{V}$ on $(0, b]$. To show there are no conjugate points of $(W, v(t) I,\{0\}, 0)$ on $(0, b]$ it suffices to show there are no conjugate points of $(R, v(t) I,\{0\}, 0)$ on $(0, b]$ where $R$ is the real line and $I$ again is the identity. But we can compare $(R, v(t) I,\{0\}, 0)$ with ' $\mathscr{V}$ and since $1 \leqq(' d-1)$ use the first part of the proof to conclude there are no conjugate points of $(R, v(t) I,\{0\}, 0)$ on $(0, b]$. q.e.d.

Comparison TheOrem 3.3. Let $\mathscr{V}$ and ' $\mathscr{V}$ be as above. Assume for each $t \in[0, b]$ the maximum eigenvalue of $R_{t}$ is $\leqq$ the minimum eigenvalue of ' $R_{t}$ and assume the minimum eigenvalue of $S$ is $\geqq$ the maximum eigenvalue of ' $S$. Assume either of the following:

(a) let $p={ }^{\prime} p=0$, let $X \in \mathscr{L}(V, b,\{0\})$ and $Y \in \mathscr{L}\left({ }^{\prime} V, b,\{0\}\right)$ be Jacobi fields with $\|\nabla X(0)\|=\|\nabla Y(0)\| \neq 0$, and assume there are no conjugate points of ' $\mathscr{V}$ on $(0, b]$; or

(b) let $p>0$ and ' $p={ }^{\prime} d-1$, let $X \in \mathscr{L}(V, b, K)$ and $Y \in \mathscr{L}\left({ }^{\prime} V, b,{ }^{\prime} K\right)$ be $K$ and ' $K$ Jacobi fields respectively such that $\|X(0)\|=\|Y(0)\| \neq 0$, and assume there are no focal points of ' $\mathscr{V}$ on $(0, b]$.

Then $\|X(t)\| \geqq\|Y(t)\|$ for $t \in[0, b]$. Moreover, if $\left\|X\left(t_{0}\right)\right\|=\left\|Y\left(t_{0}\right)\right\|$ for some $t_{0} \in[0, b]$, then $\|X(t)\|=\|Y(t)\|$ for $t \in\left[0, t_{0}\right]$.

Remark. Part (a) is the Rauch comparison theorem except for differences of notation and for our extension to the case where $\operatorname{dim} V \neq \operatorname{dim}{ }^{\prime} V$. Part (b) is the extension we will need for submanifolds. The proof is that given by Rauch except for the obvious generalizations needed for part (b) and for the technicality introduced by allowing the dimensions of $V$ and ' $V$ to be different. We again treat both cases at the same time, observing that in case (a), $K=\{0\},{ }^{\prime} K=\{0\}$ and the assumptions on $S$ and ' $S$ are vacuous.

Proof. Let

$$
\begin{aligned}
& f_{1}(t)=\langle X(t), X(t)\rangle, \\
& f_{2}(t)=\langle Y(t), Y(t)\rangle .
\end{aligned}
$$

To prove the theorem we will apply the following lemma.

LEMMA 3.4. Let $f_{1}$ and $f_{2}$ be real valued functions on $[0, b]$ satisfying

(1) $f_{1}$ and $f_{2}$ are differentiable of class $C^{1}$,

(2) $f_{1}>0$ and $f_{2}>0$ on $(0, b]$,

(3) $\lim _{t \rightarrow 0}\left(f_{1}(t) / f_{2}(t)\right)=1$,

(4) $\left(f_{1}{ }^{\prime}(t) / f_{1}(t)\right) \geqq\left(f_{2}{ }^{\prime}(t) / f_{2}(t)\right)$ on $(0, b]$. 
Then $f_{1}(t) \geqq f_{2}(t)$ on $[0, b]$. Moreover, if $f_{1}\left(t_{0}\right)=f_{2}\left(t_{0}\right)$ for some $t_{0} \in[0, b]$, then $f_{1}(t) \equiv f_{2}(t)$ on $\left[0, t_{0}\right]$.

Proof of lemma. Let $g_{i}=f_{i}^{\prime} \mid f_{i}$ on $(0, b]$ for $i=1,2$. Let $0<t_{1} \leqq t \leqq b$ It follows from integrating and taking exponentials that

$$
f_{i}(t) / f_{i}\left(t_{1}\right)=\exp \left(\int_{t_{1}}^{t} g_{i} d t\right)
$$

Hence

$$
f_{1}(t) / f_{2}(t)=\left(f_{1}\left(t_{1}\right) / f_{2}\left(t_{1}\right)\right) \exp \left(\int_{t_{1}}^{t}\left(g_{1}-g_{2}\right) d t\right) .
$$

Let $t_{1} \rightarrow 0$. Then

$$
f_{1}(t) / f_{2}(t)=\exp \left(\int_{0}^{t}\left(g_{1}-g_{2}\right) d t\right) .
$$

By assumption, $\left(g_{1}-g_{2}\right) \geqq 0$ on $(0, b]$. Hence

$$
f_{1}(t) \geqq f_{2}(t) \quad \text { on }[0, b] .
$$

Now suppose $f_{1}\left(t_{0}\right)=f_{2}\left(t_{0}\right)$ for some $t_{0} \in[0, b]$. Then

$$
\exp \left(\int_{0}^{t_{0}}\left(g_{1}-g_{2}\right) d t\right)=1,
$$

which implies $g_{1} \equiv g_{2}$ on $\left(0, t_{0}\right]$. And this implies

$$
f_{1}(t) \equiv f_{2}(t) \quad \text { on }\left[0, t_{0}\right] \text {. q.e.d. }
$$

We return to the proof of the theorem. Observe that according to Theorem 3.2 there are no focal points of $\mathscr{V}$ on $(0, b]$. To prove the theorem we need only verify that the hypotheses of the lemma are satisfied. (1) and (2) are obvious and (3) follows immediately from the initial conditions. We prove (4). Assume first that $d \leqq ' d$. Let $t_{1} \in(0, b]$. We must show

$$
\left(f_{1}^{\prime}\left(t_{1}\right) / f_{1}\left(t_{1}\right)\right) \geqq\left(f_{2}^{\prime}\left(t_{1}\right) / f_{2}\left(t_{1}\right)\right) \text {. }
$$

Let

$$
W(t)=X(t) /\left\|X\left(t_{1}\right)\right\|
$$

and

$$
Z(t)=Y(t) /\left\|Y\left(t_{1}\right)\right\|
$$

Then we must show

$$
\langle W, W\rangle^{\prime}\left(t_{1}\right) \geqq\langle Z, Z\rangle^{\prime}\left(t_{1}\right),
$$


and since $W$ and $Z$ are $K$ - and ' $K$-Jacobi fields, respectively, this is equivalent with showing

$$
I_{\left(t_{1}, K\right)}(W, W) \geqq I_{\left(t_{1}, ' K\right)}(Z, Z) .
$$

Let $e_{1}, \cdots, e_{d-1}$ be an orthonormal basis of $V$ such that $W\left(t_{1}\right)=e_{1}$. Let $f_{1}, \cdots, f_{d-1}$ be an orthonormal basis of ' $V$ such that $Z\left(t_{1}\right)=f_{1}$.

$$
W(t)=\sum_{i=1}^{d-1} g_{i}(t) e_{i}
$$

Let

$$
\tilde{W}(t)=\sum_{i=1}^{d-1} g_{i}(t) f_{i}
$$

Then $\tilde{W} \in \mathscr{L}\left({ }^{\prime} V, t_{1},{ }^{\prime} K\right)$. (In case (b) this is because ' $K={ }^{\prime} V$.) Moreover $\tilde{W}\left(t_{1}\right)=Z\left(t_{1}\right)$. Hence

$$
I_{\left(t_{1}, K\right)}(W, W) \geqq I_{\left(t_{1},{ }^{\prime} K\right)}(\tilde{W}, \tilde{W}) \geqq I_{\left(t_{1},{ }^{\prime} K\right)}(Z, Z) .
$$

The second equality follows from the minimization theorem and the first follows the hypotheses on $R_{t}$, ' $R_{t}, S$ and ' $S$, and the fact that $\|\tilde{W}(t)\|=\|W(t)\|$ and $\|\nabla \tilde{W}(t)\|=\|\nabla W(t)\|$.

Now suppose $d>^{\prime} d$. Let $W$ be a $\left(d-{ }^{\prime} d\right)$-dimensional real inner product space and let

$$
{ }^{\prime} V={ }^{\prime} V \oplus W
$$

with the induced inner product. Let $v(t)$ be the minimum eigenvalue of ' $R_{t}$ and let

$$
\text { " } R_{t}={ }^{\prime} R_{t} \oplus v(t) I
$$

where $I$ is the identity on $W$. In case (a) let " $K=\{0\}$ and " $S=0$. In case (b) let " $K={ }^{\prime \prime} V$ and let

$$
\text { " } S=' S \oplus \eta I
$$

where $\eta$ is the maximum eigenvalue of ' $S$ and $I$ is again the identity on $W$. Finally, let

$$
\text { "थ }=\left(" V, " R_{t}, " V, " S\right)
$$

Observe that the first focal point for " $\mathscr{V}$ is either the first focal point for ' $\mathscr{V}$ or the first focal point for $(W, v(t) I,\{0\}, 0)$ in case (a) and $(W, v(t) I, W, \eta I)$ in case (b). By assumption, ' $\mathscr{V}$ has no focal points on $(0, b]$ and it follows from Theorem 3.2 by comparing $(W, v(t) I,\{0\}, 0)$ (or $(W, v(t) I, W, \eta I))$ with ' $V$ that there are no focal points of $(W, v(t) I,\{0\}, 0)$ (or $(W, v(t) I, W, \eta I))$ on $(0, b]$. Hence there are no focal points of " $\mathscr{V}$ on $(0, b]$, and the hypotheses of the theorem are satisfied for $\mathscr{V}$ and " $\mathscr{V}$. Since now $\operatorname{dim} V=\operatorname{dim} " V$, the theorem holds for $\mathscr{V}$ and $\mathscr{V}$ by the first part 
of the proof. We apply this to prove the theorem for $\mathscr{V}$ and ' $\mathscr{V}$. Simply observe that in case (a) if $Y(t) \in \mathscr{L}\left({ }^{\prime} V, b,\{0\}\right)$ is a Jacobi field, the curve

$$
(Y(t), 0) \in \mathscr{L}(" V, b,\{0\})
$$

is again a Jacobi field with initial derivative the same length and with

$$
\|Y(t)\|=\|(Y(t), 0)\| \text {. }
$$

Similarly in case (b), if $Y(t) \in \mathscr{L}\left({ }^{\prime} V, b,{ }^{\prime} K\right)$ is a ' $K$-Jacobi field, then

$$
(Y(t), 0) \in \mathscr{L}\left(" V, b,{ }^{\prime \prime} K\right)
$$

is a "K-Jacobi field with

$$
\|Y(t)\|=\|(Y(t), 0)\| \text {. q.e.d. }
$$

4. Applications to submanifolds. We adopt the notation and geometrical setups of $\S 2$. So $M$ is a $d$-dimensional Riemannian manifold, $d \geqq 2, K$ a $p$-dimensional Riemannian submanifold of $M$ with $0 \leqq p \leqq d-1, m$ a point on $K, \sigma(t)$ a geodesic in $M$ parametrized by arc length with domain the interval $[0, b]$, initial point $m$ and initial derivative $\sigma_{*}(0) \in\left(K_{m}\right)^{\perp}$. And ' $M,{ }^{\prime} K,{ }^{\prime} \sigma$ denotes another such setup, with $\operatorname{dim}^{\prime} M={ }^{\prime} d \geqq 2, \operatorname{dim}^{\prime} K={ }^{\prime} p$ with $0 \leqq{ }^{\prime} p \leqq ' d-1$ and domain of ' $\sigma$ the interval $[0, b]$.

We will make the same assumption on the sectional curvatures and second fundamental forms several times, so for convenience we abbreviate them. Assumptions (K) and $(\mathrm{S})$ will mean the following:

(K) For each $t \in[0, b]$ and for all 2-planes $P \subset M_{\sigma(t)}$ containing $\sigma_{*}(t)$ and all 2-planes $Q \subset{ }^{\prime} M_{\sigma(t)}$ containing ' $\sigma_{*}(t)$ the sectional curvatures $K(P)$ and $K(Q)$ satisfy:

$$
K(P) \leqq K(Q) .
$$

(S) The minimum eigenvalue of $S$ is $\geqq$ the maximum eigenvalue of ' $S$.

Observe that $(\mathrm{K})$ is equivalent with the following. For each $t$ restrict the curvature transformation $R_{t}$ to the subspace $\left(M_{\sigma(t)}\right)^{\perp}$ of $M_{\sigma(t)}$ orthogonal to $\sigma_{*}(t)$ (similarly for ' $R_{t}$ ). Then, for example, the maximum sectional curvature $K(P)$ as $P$ runs over the 2-planes in $M_{\sigma(t)}$ containing $\sigma_{*}(t)$ equals the maximum of $\left\langle R_{t} x, x\right\rangle$ as $x$ runs over the unit sphere in $\left(M_{\sigma(t)}\right)^{\perp}$. So $(\mathrm{K})$ is equivalent with the assumption that the maximum eigenvalue of $R_{t}$ is $\leqq$ the minimum eigenvalue of ' $R_{t}$ for each $t \in[0, b]$.

The following theorem describing the relative location of first focal points along $\sigma$ and ' $\sigma$ is now an immediate application of Theorem 3.2.

THEOREM 4.1. Assume that if $p>0$ then ' $p>0$. Assume (K) and (S). (Note (S) vacuous when $p=0$.) Then if there are no focal points on ' $\sigma$, there are no focal points on $\sigma$. 
REMARK. Some of the most useful comparison spaces are the manifolds of constant curvature. Let $c$ and $\delta$ be any real numbers. Let $M(c)$ be a complete Riemannian manifold of $\operatorname{dim} \geqq 2$ and with constant curvature $c$. Let $p \in M(c)$ and let $v$ be a unit vector in $(M(c))_{p}$. Choose a hypersurface $N$ in $M(c)$ through $p$ such that $v \in\left(N_{p}\right)^{\perp}$ and such that all the eigenvalues of the second fundamental form $S_{v}$ are equal to $\delta$. Let $\tau$ be the geodesic in $M(c)$ with $\tau(0)=p$ and $\tau_{*}(0)=v$. Let $t_{0}$ be the smallest positive solution of

$$
\begin{aligned}
\operatorname{ctn} c^{1 / 2} t & =-\delta / c^{1 / 2} & & \text { if } c>0 \\
t & =-1 / \delta & & \text { if } c=0 \\
\operatorname{coth} c^{1 / 2} t & =-\delta / c^{1 / 2} & & \text { if } c<0 .
\end{aligned}
$$

Then the first focal point to $N$ along $\tau$ occurs at $\tau\left(t_{0}\right)$.

Corollary 4.2. Let $M, K, \sigma$ be as above. Assume $p>0$. Let $c$ and $\delta$ be real numbers and define $t_{0}$ as above.

(a) Assume all the eigenvalues of $S_{\sigma_{*}(0)}$ are $\geqq \delta$ and the sectional curvatures of all 2-planes along $\sigma$ containing $\sigma_{*}$ are all $\leqq c$. Then there are no focal points of $K$ on $\sigma \mid\left[0, t_{0}\right)$.

(b) If all the eigenvalues of $S_{\sigma_{*}(0)}$ are $\leqq \delta$ and the sectional curvatures of all 2 -planes along $\sigma$ containing $\sigma_{*}$ are all $\geqq c$, then there is a focal point of $K$ on $\sigma \mid\left[0, t_{0}\right]$.

This corollary was proved by Hermann in [4] in a somewhat different fashion. The following theorem follows directly from Theorem 3.3. Part (a) is the Rauch comparison theorem and part (b) gives a comparison in the case ' $K$ is a hypersurface, i.e., $\operatorname{dim}^{\prime} K={ }^{\prime} d-1$. We do not assume $d={ }^{\prime} d$.

THEOREM 4.3. Assume either of the following:

(a) let $X \in \mathscr{L}(\sigma, b, 0)$ and $Y \in \mathscr{L}\left({ }^{\prime} \sigma, b, 0\right)$ be Jacobi fields such that $\|\nabla X(0)\|=\|\nabla Y(0)\|$, assume $(\mathrm{K})$ and assume there are no conjugate points on ' $\sigma$; or

(b) let ' $p=$ ' -1 , let $X \in \mathscr{L}(\sigma, b, K)$ and $Y \in \mathscr{L}\left({ }^{\prime} \sigma, b,{ }^{\prime} K\right)$ be $K$ - and 'KJacobi fields respectively such that $\|X(0)\|=\|Y(0)\| \neq 0$, assume (K) and (S) and assume there are no focal points on ' $\sigma$.

Then $\|X(t)\| \geqq\|Y(t)\|$ for $t \in[0, b]$. Moreover, if $\left\|X\left(t_{0}\right)\right\|=\left\|Y\left(t_{0}\right)\right\|$ for some $t_{0} \in[0, b]$, then $\|X(t)\| \equiv\|Y(t)\|$ on $\left[0, t_{0}\right]$.

Observe that in part (b) of the theorem, since ' $K$ is assumed to be a hypersurface, ' $K$-Jacobi fields are automatically strong ' $K$-Jacobi fields. In the general case when ' $K$ is not a hypersurface we will have to restrict to the strong ' $K$-Jacobi fields to get a comparison. ' $K$-Jacobi fields which are not strong we call weak. The following example shows part (b) of the theorem cannot hold in the case ' $p<$ ' $d-1$ if we allow $Y$ to be a weak ' $K$-Jacobi field. 
EXAMPLE 1. Let $\left(e_{1}(t), e_{2}(t), \sigma_{*}(t)\right)$ be a parallel orthonormal frame along a geodesic $\sigma$ (parametrized by arc length) on the 3 -sphere $S^{3}$ of constant curvature 1. Let $\tau$ be the geodesic through $\sigma(0)$ with $\tau_{*}(0)=e_{1}(0)$. Let $X(t)=(\cos t) e_{1}(t)$ and $Y(t)=(\cos t) e_{1}(t)+(\sin t) e_{2}(t)$. Then $X$ and $Y$ are both $\tau$-Jacobi fields along $\sigma$. Moreover, $Y$ is a weak $\tau$-Jacobi field. $\|X(0)\|=\|Y(0)\| \neq 0$, and (K) and (S) hold trivially, but $\|X(t)\|<\|Y(t)\|$ for $0<t<\pi$.

Now let $0<{ }^{\prime} p<{ }^{\prime} d-1$. We will get a comparison of $K$-Jacobi fields with strong ' $K$-Jacobi fields essentially by enlarging ' $K$ to a hypersurface $\widetilde{K}$ in such a way that strong ' $K$-Jacobi fields become $\widetilde{K}$-Jacobi fields; then we apply Theorem 4.3 to $K$ and $\widetilde{K}$. This will yield a comparison of $K$ and strong ' $K$-Jacobi fields, but only as far out as the first focal point for $\tilde{K}$ which will occur at least as soon as the first focal point for ' $K$. This, however, is the best one can expect-we give an example later of a $K$-Jacobi field and a strong ' $K$-Jacobi field for which the comparison holds as far as the first $\tilde{K}$ focal point but not as far as the first ' $K$ focal point.

Let $H$ be the orthogonal complement of ${ }^{\prime} K_{\prime^{\prime}}$ in $\left({ }^{\prime} M^{\prime} \sigma(0)\right)^{\perp}$. Let $\eta$ be the maximum eigenvalue of $S_{\sigma_{*}(0)}$. Choose a hypersurface $\widetilde{K}$ through ' $m$ orthogonal to ${ }^{\prime} \sigma_{*}(0)$ (i.e., so that $\left.\tilde{K}_{{ }^{\prime}}=\left({ }^{\prime} M_{\sigma^{\prime}(0)}\right)^{\perp}\right)$ so that the second fundamental form

$$
\tilde{S}_{\sigma_{*}(0)}=S_{\sigma_{*}(0)} \oplus \eta I,
$$

where $I$ is the identity on $H$. This is possible in view of Theorem 3, page 198 of [3]. Since $\tilde{S}_{\sigma_{*}(0)} \mid{ }^{\prime} K_{\prime_{m}}=S_{\sigma_{*}(0)}$, strong ' $K$-Jacobi fields are also (strong) $\widetilde{K}$-Jacobi fields. By applying Theorem 4.3 to $K$ and $\widetilde{K}$ we obtain the following comparison of $K$ and strong ' $K$ Jacobi fields.

TheOREM 4.4. Let $0<{ }^{\prime} p<{ }^{\prime} d-1$. Let $X \in \mathscr{L}(\sigma, b, K)$ be a K-Jacobi field and $Y \in \mathscr{L}\left({ }^{\prime} \sigma, b,{ }^{\prime} K\right)$ a strong ' $K$-Jacobi field such that $\|X(0)\|=\|Y(0)\| \neq 0$. Assume (K) and (S) and assume there are no focal points of $\tilde{K}$ on ' $\sigma$. Then $\|X(t)\| \geqq\|Y(t)\|$ for $t \in[0, b]$. Moreover, if $\left\|X\left(t_{0}\right)\right\|=\left\|Y\left(t_{0}\right)\right\|$ for some $t_{0} \in[0, b]$, then $\|X(t)\| \equiv\|Y(t)\|$ on $\left[0, t_{0}\right]$.

REMARK 1. The first focal point for $\tilde{K}$ along ' $\sigma$ occurs at least as soon as the first focal point for ' $K$ along ' $\sigma$. For suppose $\sigma\left(t_{1}\right)$ is a focal point for ' $K$. Then there is a nonzero vector field $Z \in \mathscr{L}\left({ }^{\prime} \sigma, t_{1},{ }^{\prime} K\right)$ vanishing at $t_{1}$ for which $I_{\left(t_{1},{ }^{\prime} K\right)}(Z, Z)=0$. But $I_{\left(t_{1},{ }^{\prime} K\right)}(Z, Z)=I_{\left(t_{1}, \tilde{\mathbf{k}}\right)}(Z, Z)$ and $Z \in \mathscr{L}\left({ }^{\prime} \sigma, t_{1}, \tilde{K}\right)$. Therefore $\left.I_{\left(t_{1}\right.} \tilde{K}\right)$ is not positive definite on the subspace of vector fields in $\mathscr{L}\left({ }^{\prime} \sigma, t_{1}, \widetilde{K}\right)$ vanishing at $t_{1}$. Hence there is a focal point of $\tilde{K}$ on ' $\sigma$ prior to (or at) $\sigma\left(t_{1}\right)$. Our choice, however, of $\tilde{K}$ was the most economical one in the sense that among all such hypersurfaces whose second fundamental forms agree with $S_{\sigma_{*}(0)}$ on ' $K_{\prime} m$, $\tilde{K}$ is the one whose first focal point occurs farthest along ' $\sigma$.

REMARK 2. We give a crude estimate of the location of the first focal point for $\tilde{K}$. Let $c$ be the sup of the sectional curvatures of all the plane sections along ' $\sigma$ 
containing ' $\sigma_{*}$. Let $\delta$ be the minimum eigenvalue of $S_{\sigma_{*}(0)}$. Let $t_{0}$ be the smallest positive solution of

$$
\begin{aligned}
\operatorname{ctn} c^{1 / 2} t & =-\delta / c^{1 / 2} & & \text { if } c>0, \\
t & =-1 / \delta & & \text { if } c=0, \\
\operatorname{coth} c^{1 / 2} t & =-\delta / c^{1 / 2} & & \text { if } c<0 .
\end{aligned}
$$

Then, by Corollary 4.2, the first focal point for $\tilde{K}$ does not occur prior to ' $\sigma\left(t_{0}\right)$.

As we have mentioned, the comparison in Theorem 4.4 does not in general hold as far as the first focal point for ' $K$. Consider the following example.

EXAMPLE 2. Let $M=S^{4}$, the 4-sphere with constant curvature equal to 1 . Let $\left(e_{1}(t), e_{2}(t), e_{3}(t), \sigma_{*}(t)\right)$ be a parallel orthonormal frame along a geodesic $\sigma$ on $S^{4}$. Let the submanifold $K$ be the geodesic through $\sigma(0)$ tangent to $e_{1}(0)$. So the second fundamental form $S_{\sigma_{*}(0)} \equiv 0$. Let

$$
X(t)=\left((101)^{1 / 2} \cos t\right) e_{1}(t) .
$$

Then $X$ is a (strong) $K$-Jacobi field along $\sigma$. Let ${ }^{\prime} M=P^{4}(C)$, the four dimensional complex projective space with curvature normalized so that the maximum sectional curvature is 4 . Let $\left(f_{1}(t), f_{2}(t), f_{3}(t),{ }^{\prime} \sigma_{*}(t)\right)$ be a parallel orthonormal frame along a geodesic ' $\sigma$ on $P^{4}(C)$ chosen so as to simultaneously diagonalize the curvature transformations ' $R_{t}$. We can assume the sectional curvatures of the plane sections spanned by $f_{1}$ and ' $\sigma_{*}$ are equal to 4 . Then the plane sections spanned by $f_{2}$ or $f_{3}$ and ' $\sigma_{*}$ have curvature 1 . Let the submanifold ' $K$ be the geodesic through ' $\sigma(0)$ tangent to $f_{1}(0)+10 f_{2}(0)$. The second fundamental form $S_{\sigma_{*}(0)} \equiv 0$. Let

$$
Y(t)=(\cos 2 t) f_{1}(t)+(10 \cos t) f_{2}(t) .
$$

Then $Y$ is a strong ' $K$-Jacobi field along ' $\sigma$. Moreover $\|X(0)\|=\|Y(0)\|$, and in this example we even have $\|\nabla X(0)\|=\|\nabla Y(0)\|=0$. According to the theorem the length of $X$ should dominate the length of $Y$ prior to the first focal point for $\tilde{K}$. $\widetilde{K}$ in this case is a hypersurface through ' $\sigma(0)$ orthogonal to ' $\sigma_{*}(0)$ and totally geodesic at $\sigma(0)$, i.e. $\tilde{S}_{\sigma_{+}(0)} \equiv 0$. The first focal point for $\tilde{K}$ occurs at ${ }^{\prime} \sigma(\pi / 4)$. It is easy to check that $\|X(t)\| \geqq\|Y(t)\|$ for $t<(\pi / 3)$. At $\pi / 3$ the lengths become equal, and beyond $\pi / 3$ the length of $Y$ becomes greater than the length of $X$. Let

and

$$
Y_{1}(t)=(-5 \sin 2 t) f_{1}(t)+(\sin t) f_{2}(t)
$$

$$
Y_{2}(t)=(\sin t) f_{3}(t) .
$$

Then $Y, Y_{1}, Y_{2}$ form a basis of the ' $K$-Jacobi fields along ' $\sigma$. The first focal point of ' $K$ occurs when these vector fields first become linearly dependent. It is easy to compute that this first focal point for ' $K$ occurs at ${ }^{\prime} \sigma\left(t_{0}\right)$ where $t_{0}$ is the smallest positive solution of 


$$
\cos ^{2} t=1 / 102 \text {. }
$$

So the first focal point for ' $K$ occurs beyond ' $\sigma(4 \pi / 9)$.

There are certain special cases where the comparison in Theorem 4.4 holds out to and sometimes beyond the first focal point for ' $K$.

Definition. We say the Jacobi equation splits along ' $\sigma$ relative to ' $K$ if the curvature transformations ${ }^{\prime} R_{t}$ preserve ${ }^{\prime} K_{{ }_{m}}$ and the parallel translates of ${ }^{\prime} K_{{ }_{m}}$ along ' $\sigma$.

Definition. A point ' $\sigma\left(t_{0}\right), t_{0} \neq 0$, on ' $\sigma$ is a strong focal point of ' $K$ if there exists a nontrivial strong ' $K$-Jacobi field vanishing at ' $\sigma\left(t_{0}\right)$.

Suppose the Jacobi equation splits along ' $\sigma$ relative to ' $K$. Restrict the curvature transformation ' $R_{t}$ to $\left({ }^{\prime} M_{\sigma(t)}\right)^{\perp}$, the subspace of ' $M_{\sigma(t)}$ orthogonal to ' $\sigma_{*}(t)$. Let $V_{t}^{1}$ be the parallel translate of ' $K_{m}$ along ' $\sigma$ to ' $\sigma(t)$, and let $V_{t}^{2}$ be the orthogonal complement to $V_{t}^{1}$ in $\left({ }^{\prime} M^{\prime}{ }_{\sigma(t)}\right)^{\perp}$. By assumption, ' $R_{t}$ preserves $V_{t}^{1}$. Since ' $R_{t}$ is symmetric, it also preserves $V_{t}^{2}$. Let ' $R_{t}{ }^{1}={ }^{\prime} R_{t} \mid V_{t}{ }^{1}$ and ${ }^{\prime} R_{t}{ }^{2}={ }^{\prime} R_{t} \mid V_{t}{ }^{2}$. Then

$$
{ }^{\prime} R_{t}={ }^{\prime} R_{t}^{1} \oplus{ }^{\prime} R_{t}^{2} \text {. }
$$

Let $Y$ be a Jacobi field along ' $\sigma$ everywhere orthogonal to ' $\sigma_{*}$, and let $Y(t)=Y_{1}(t)+Y_{2}(t)$ where $Y_{1}(t) \in V_{t}^{1}$ and $Y_{2}(t) \in V_{t}^{2}$. Then $Y_{1}$ and $Y_{2}$ are Jacobi fields along ' $\sigma$ and the Jacobi equation becomes:

$$
\begin{aligned}
& \left(\nabla^{2} Y_{1}\right)(t)+{ }^{\prime} R_{t}^{1}\left(Y_{1}(t)\right)=0 \\
& \left(\nabla^{2} Y_{2}\right)(t)+{ }^{\prime} R_{t}^{2}\left(Y_{2}(t)\right)=0 .
\end{aligned}
$$

If $Y$ is a ' $K$-Jacobi field along ' $\sigma$, then $Y_{1}$ is a strong ' $K$-Jacobi field and $Y_{2}$ is a $K$-Jacobi field with $Y_{2}(0)=0$ and $\nabla Y_{2}(0) \in\left({ }^{\prime} K_{m}^{\prime}\right)^{\perp}$. Moreover

$$
\left\langle Y_{1}(t), Y_{2}(t)\right\rangle \equiv 0 \text {. }
$$

If $Y$ is a strong ' $K$-Jacobi field, then $Y=Y_{1}$.

Note that in general (if the Jacobi equation is not necessarily split along ' $\sigma$ ), if $Y$ is a ' $K$-Jacobi field, $Y$ has a unique decomposition into $Y_{1}+Y_{2}$ where $Y_{1}$ is a strong ' $K$-Jacobi field and $Y_{2}$ is a ' $K$-Jacobi field vanishing at 0 . Generally $\left\langle Y_{1}(t), Y_{2}(t)\right\rangle \neq 0$. In case the Jacobi equation does split relative to ' $K$ this decomposition agrees with the one given above.

THEOREM 4.5. Let $0<{ }^{\prime} p<' d-1$. Let $X \in \mathscr{L}(\sigma, b, K)$ be a K-Jacobi field and $Y \in \mathscr{L}\left({ }^{\prime} \sigma, b,{ }^{\prime} K\right)$ a strong 'K-Jacobi field such that $\|X(0)\|=\|Y(0)\| \neq 0$. Assume (K) and (S), assume the Jacobi equation splits along ' $\sigma$ relative to ' $K$, and assume there are no strong focal points of 'Kon' $\sigma$. Then $\|X(t)\| \geqq\|Y(t)\|$ for $t \in[0, b]$. Moreover, if $\left\|X\left(t_{0}\right)\right\|=\left\|Y\left(t_{0}\right)\right\|$ for some $t_{0} \in[0, b]$, then $\|X(t)\| \equiv\|Y(t)\|$ on $\left[0, t_{0}\right]$.

Proof. The proof is again an application of Theorem $3.3 \mathrm{~b}$, with the difference 
that in this case we define ' $\mathscr{V}\left({ }^{\prime} V,{ }^{\prime} R_{t},{ }^{\prime} K,{ }^{\prime} S\right)$ as follows. Let ' $V={ }^{\prime} K_{m}$, so in this case $\operatorname{dim}^{\prime} V={ }^{\prime} p$. The symmetric linear transformations ' $R_{t}$ on ' $V$ are in this case to equal $\left.P_{t}^{-1} \circ{ }^{\prime} R_{t}^{1} \circ P^{t}\right|^{\prime} K_{\prime_{m}}$, where $P_{t}$ is parallel translation along ' $\sigma$. Finally ' $K={ }^{\prime} V$ and ' $S=S_{\sigma_{*}(0)}$. The assumption that there are no strong focal points of ' $K$ on ' $\sigma$ means that there are no focal points of ' $\mathscr{V}$ on $(0, b]$. Since $Y$ is a strong ' $K$-Jacobi field, if we collapse $Y$ along ' $\sigma$ via parallel translation we obtain a curve in ' $V$ which is a ' $K$-Jacobi field (in the sense of ' $\mathscr{V}$ ).

q.e.d.

Remark. Theorem 4.5 does not use the full force of assumption (K). Since the Jacobi equation is split along ' $\sigma$ we need only assume $(\mathrm{K})$ holds for the sectional curvatures of those plane sections determined by elements of $V_{l}{ }^{1}$ (the parallel translate of ${ }^{\prime} K_{m}$ along ' $\sigma$ ) and ${ }^{\prime} \sigma_{*}(t)$.

Corollary 4.6. Let $0<{ }^{\prime} p<' d-1$. Let $X \in \mathscr{L}(\sigma, b, K)$ be a $K$-Jacobi field and $Y \in \mathscr{L}\left({ }^{\prime} \sigma, b,{ }^{\prime} K\right) a^{\prime} K$-Jacobi field such that $\|X(0)\|=\|Y(0)\| \neq 0 . Y=Y_{1}+Y_{2}$ where $Y_{1}$ is a strong ' $K$-Jacobi field and $Y_{2}(0)=0$. Assume $(\mathrm{K})$ and $(\mathrm{S})$, assume the Jacobi equation splits along ' $\sigma$ relative to ' $K$, and assume there are no strong focal points of ' $K$ on ' $\sigma$. Then

$$
\langle X(t), X(t)\rangle \geqq\langle Y(t), Y(t)\rangle-\left\langle Y_{2}(t), Y_{2}(t)\right\rangle
$$

for $t \in[0, b]$. Moreover, if equality occurs for some $t_{0}$, then equality holds on $\left[0, t_{0}\right]$.

Proof. By Theorem 4.5. $\langle X(t), X(t)\rangle \geqq\left\langle Y_{1}(t), Y_{1}(t)\right\rangle$ for $t \in[0, b]$. But $\left\langle Y_{1}, Y_{1}\right\rangle=\langle Y, Y\rangle-\left\langle Y_{2}, Y_{2}\right\rangle$ since $\left\langle Y_{1}, Y_{2}\right\rangle \equiv 0$ along ' $\sigma$. $\quad$ q.e.d.

Corollary 4.7. Let $0<p<d-1$ and $0<{ }^{\prime} p<{ }^{\prime} d-1$. Let $X \in \mathscr{L}(\sigma, b, K)$ be a $K$-Jacobi field and $Y \in \mathscr{L}\left(' \sigma, b,{ }^{\prime} K\right)$ a 'K-Jacobi field. Let $X=X_{1}+X_{2}$ and $Y=Y_{1}+Y_{2}$ where $X_{1}$ is a strong $K$-Jacobi field, $Y_{1}$ a strong ' $K$-Jacobi field, $X_{2}(0)=0$, and $Y_{2}(0)=0$. Assume $\|X(0)\|=\|Y(0)\| \neq 0$ and $\left\|\nabla X_{2}(0)\right\|$ $=\left\|\nabla Y_{2}(0)\right\|$. Assume $(\mathrm{K})$ and $(\mathrm{S})$, assume the Jacobi equation splits both along $\sigma$ relative to $K$ and along ' $\sigma$ relative to ' $K$, and assume that there are no conjugate points on ' $\sigma$ and no strong focal points of ' $K$ on ' $\sigma$. Then $\|X(t)\| \geqq\|Y(t)\|$ for $t \in[0, b]$. Moreover, if $\left\|X\left(t_{0}\right)\right\|=\left\|Y\left(t_{0}\right)\right\|$ for some $t_{0} \in[0, b]$ then $\|X(t)\|$ $\equiv\|Y(t)\|$ on $\left[0, t_{0}\right]$.

Proof. It follows from Theorem 4.5 that $\left\|X_{1}(t)\right\| \geqq\left\|Y_{1}(t)\right\|$ on $[0, b]$ and from Theorem 4.3a that $\left\|X_{2}(t)\right\| \geqq\left\|Y_{2}(t)\right\|$ on $[0, b]$. Since the Jacobi equation is split along both geodesics $\left\langle X_{1}, X_{2}\right\rangle \equiv 0$ and $\left\langle Y_{1}, Y_{2}\right\rangle \equiv 0$. Hence $\|X(t)\| \geqq\|Y(t)\|$ on $[0, b]$, and the equality statement follows easily.

Remark. If ' $M$ has constant curvature, the Jacobi equation always splits along ' $\sigma$ for any submanifold ' $K$. If ' $M$ is a symmetric space, the Jacobi equation splits along a geodesic ' $\sigma$ for those submanifolds ' $K$ whose tangent space ' $K_{{ }^{\prime}}$ is spanned by eigenvectors of the curvature transformation ' $R_{0}$ on $\left({ }^{\prime} M_{\sigma(0)}\right)^{\perp}$. 


\section{BIBLIOGRAPHY}

1. W. Ambrose, The Cartan structural equations in classical Riemannian geometry, J. Indian. Math. Soc. 24 (1960), 23-76.

2. M. Berger, An extension of Rauch's metric comparison theorem and some applications, Illinois J. Math. 6 (1962), 700-712.

3. R. Bishop and R. Crittenden, Geometry of manifolds, Academic Press, New York, 1964.

4. R. Hermann, Focal points of closed submanifolds of Riemannian spaces, Nederl. Akad. Wetensch. Proc. Ser. A 66 (1963), 613-628.

5. H. Rauch, A contribution to differential geometry in the large, Ann. of Math. 54 (1951), 38-55.

6. - Geodesics and curvature in differential geometry in the large, Yeshiva Univ, Press, New York, 1959.

\section{UNIVERSITY OF CALIFORNia,} Berkeley, CALIFornia 